\title{
The Veldkamp Space of Two-Qubits
}

\author{
Metod SANIGA ${ }^{\dagger}$, Michel PLANAT ${ }^{\ddagger}$, Petr PRACNA ${ }^{\S}$ and Hans HAVLICEK $₫$ \\ † Astronomical Institute, Slovak Academy of Sciences, \\ SK-05960 Tatranská Lomnica, Slovak Republic \\ E-mail: msaniga@astro.sk \\ URL: http://www.ta3.sk/ msaniga/ \\ ‡ Institut FEMTO-ST, CNRS, Département LPMO, 32 Avenue de l'Observatoire, \\ F-25044 Besançon Cedex, France \\ E-mail: michel.planat@femto-st.fr \\ $\S$ J. Heyrovsky Institute of Physical Chemistry, Academy of Sciences of the Czech Republic, \\ Dolejškova 3, CZ-182 23 Prague 8, Czech Republic \\ E-mail: pracna@jh-inst.cas.cz \\ ฯ Institut für Diskrete Mathematik und Geometrie, Technische Universität Wien, \\ Wiedner Hauptstraße 8-10, A-1040 Vienna, Austria \\ E-mail: havlicek@geometrie.tuwien.ac.at
}

Received April 13, 2007, in final form June 18, 2007; Published online June 29, 2007

Original article is available at http://www.emis.de/journals/SIGMA/2007/075/

\begin{abstract}
Given a remarkable representation of the generalized Pauli operators of twoqubits in terms of the points of the generalized quadrangle of order two, $W(2)$, it is shown that specific subsets of these operators can also be associated with the points and lines of the four-dimensional projective space over the Galois field with two elements - the so-called Veldkamp space of $W(2)$. An intriguing novelty is the recognition of (uni- and tri-centric) triads and specific pentads of the Pauli operators in addition to the "classical" subsets answering to geometric hyperplanes of $W(2)$.
\end{abstract}

Key words: generalized quadrangles; Veldkamp spaces; Pauli operators of two-qubits

2000 Mathematics Subject Classification: 51Exx; 81R99

\section{Introduction}

A deeper understanding of the structure of Hilbert spaces of finite dimensions is of utmost importance for quantum information theory. Recently, we made an important step in this respect by demonstrating that the commutation algebra of the generalized Pauli operators on the $2^{N}$-dimensional Hilbert spaces is embodied in the geometry of the symplectic polar space of rank $N$ and order two [1, 2, 3]. The case of two-qubit operator space, $N=2$, was scrutinized in very detail $[1,3]$ by explicitly demonstrating, in different ways, the correspondence between various subsets of the generalized Pauli operators/matrices and the fundamental subgeometries of the associated rank-two polar space - the (unique) generalized quadrangle of order two. In this paper we will reveal another interesting geometry hidden behind the Pauli operators of two-qubits, namely that of the Veldkamp space defined on this generalized quadrangle.

\section{$2 \quad$ Finite generalized quadrangles and Veldkamp spaces}

In this section we will briefly highlight the basics of the theory of finite generalized quadrangles [4] and introduce the concept of the Veldkamp space of a point-line incidence geometry [5] to be employed in what follows. 
A finite generalized quadrangle of order $(s, t)$, usually denoted $\mathrm{GQ}(s, t)$, is an incidence structure $S=(P, B, \mathrm{I})$, where $P$ and $B$ are disjoint (non-empty) sets of objects, called respectively points and lines, and where $\mathrm{I}$ is a symmetric point-line incidence relation satisfying the following axioms [4]: (i) each point is incident with $1+t$ lines $(t \geq 1)$ and two distinct points are incident with at most one line; (ii) each line is incident with $1+s$ points $(s \geq 1)$ and two distinct lines are incident with at most one point; and (iii) if $x$ is a point and $L$ is a line not incident with $x$, then there exists a unique pair $(y, M) \in P \times B$ for which $x \mathrm{I} M \mathrm{I} y \mathrm{I} L$; from these axioms it readily follows that $|P|=(s+1)(s t+1)$ and $|B|=(t+1)(s t+1)$. It is obvious that there exists a point-line duality with respect to which each of the axioms is self-dual. Interchanging points and lines in $S$ thus yields a generalized quadrangle $S^{D}$ of order $(t, s)$, called the dual of $S$. If $s=t, S$ is said to have order $s$. The generalized quadrangle of order $(s, 1)$ is called a grid and that of order $(1, t)$ a dual grid. A generalized quadrangle with both $s>1$ and $t>1$ is called thick.

Given two points $x$ and $y$ of $S$ one writes $x \sim y$ and says that $x$ and $y$ are collinear if there exists a line $L$ of $S$ incident with both. For any $x \in P$ denote $x^{\perp}=\{y \in P \mid y \sim x\}$ and note that $x \in x^{\perp}$; obviously, $x^{\perp}=1+s+s t$. Given an arbitrary subset $A$ of $P$, the $\operatorname{perp}\left(\right.$-set) of $A, A^{\perp}$, is defined as $A^{\perp}=\bigcap\left\{x^{\perp} \mid x \in A\right\}$ and $A^{\perp \perp}:=\left(A^{\perp}\right)^{\perp}$. A triple of pairwise non-collinear points of $S$ is called a triad; given any triad $T$, a point of $T^{\perp}$ is called its center and we say that $T$ is acentric, centric or unicentric according as $\left|T^{\perp}\right|$ is, respectively, zero, non-zero or one. An ovoid of a generalized quadrangle $S$ is a set of points of $S$ such that each line of $S$ is incident with exactly one point of the set; hence, each ovoid contains $s t+1$ points.

The concept of crucial importance is a geometric hyperplane $H$ of a point-line geometry $\Gamma(P, B)$, which is a proper subset of $P$ such that each line of $\Gamma$ meets $H$ in one or all points [6]. For $\Gamma=\mathrm{GQ}(s, t)$, it is well known that $H$ is one of the following three kinds: (i) the perp-set of a point $x, x^{\perp}$; (ii) a (full) subquadrangle of order $\left(s, t^{\prime}\right), t^{\prime}<t$; and (iii) an ovoid.

Finally, we need to introduce the notion of the Veldkamp space of a point-line incidence geometry $\Gamma(P, B), \mathcal{V}(\Gamma)[5] . \mathcal{V}(\Gamma)$ is the space in which (i) a point is a geometric hyperplane of $\Gamma$ and (ii) a line is the collection $H_{1} H_{2}$ of all geometric hyperplanes $H$ of $\Gamma$ such that $H_{1} \cap H_{2}=$ $H_{1} \bigcap H=H_{2} \bigcap H$ or $H=H_{i}(i=1,2)$, where $H_{1}$ and $H_{2}$ are distinct points of $\mathcal{V}(\Gamma){ }^{1}$ If $\Gamma=S$, from the preceding paragraph we learn that the points of $\mathcal{V}(S)$ are, in general, of three different types.

\section{The smallest thick GQ and its Veldkamp space}

The smallest thick GQ is obviously the one with $s=t=2$, dubbed the "doily." This quadrangle has a number of interesting representations of which we mention the most important two [4]. One, frequently denoted as $W_{3}(2)$ or simply $W(2)$, is in terms of the points of $P G(3,2)$ (i.e., the three-dimensional projective space over the Galois field with two elements) together with the totally isotropic lines with respect to a symplectic polarity. The other, usually denoted as $Q(4,2)$, is in terms of points and lines of a parabolic quadric in $P G(4,2)$. By abuse of notation, any GQ isomorphic to $W(2)$ will also be denoted by this symbol. From the preceding section we readily get that $W(2)$ is endowed with 15 points/lines, each line contains three points and, dually, each point is on three lines; moreover, it is a self-dual object, i.e., isomorphic to its dual. $W(2)$ features all the three kinds of hyperplanes, of the following cardinalities [5]: 15 perp-sets, $x^{\perp}$, seven points each; 10 grids (of order $(2,1)$ ), nine points each; and six ovoids, five points each - as depicted in Fig. 1. The quadrangle exhibits two distinct kinds of triads, viz. unicentric and tricentric. A point of $W(2)$ is the center of four distinct unicentric triads (Fig. 2, left); hence,

\footnotetext{
${ }^{1}$ It is important to mention here that the definition of Veldkamp space given by Shult in [7] is more restrictive than that of Buekenhout and Cohen [5] adopted in this paper.
} 

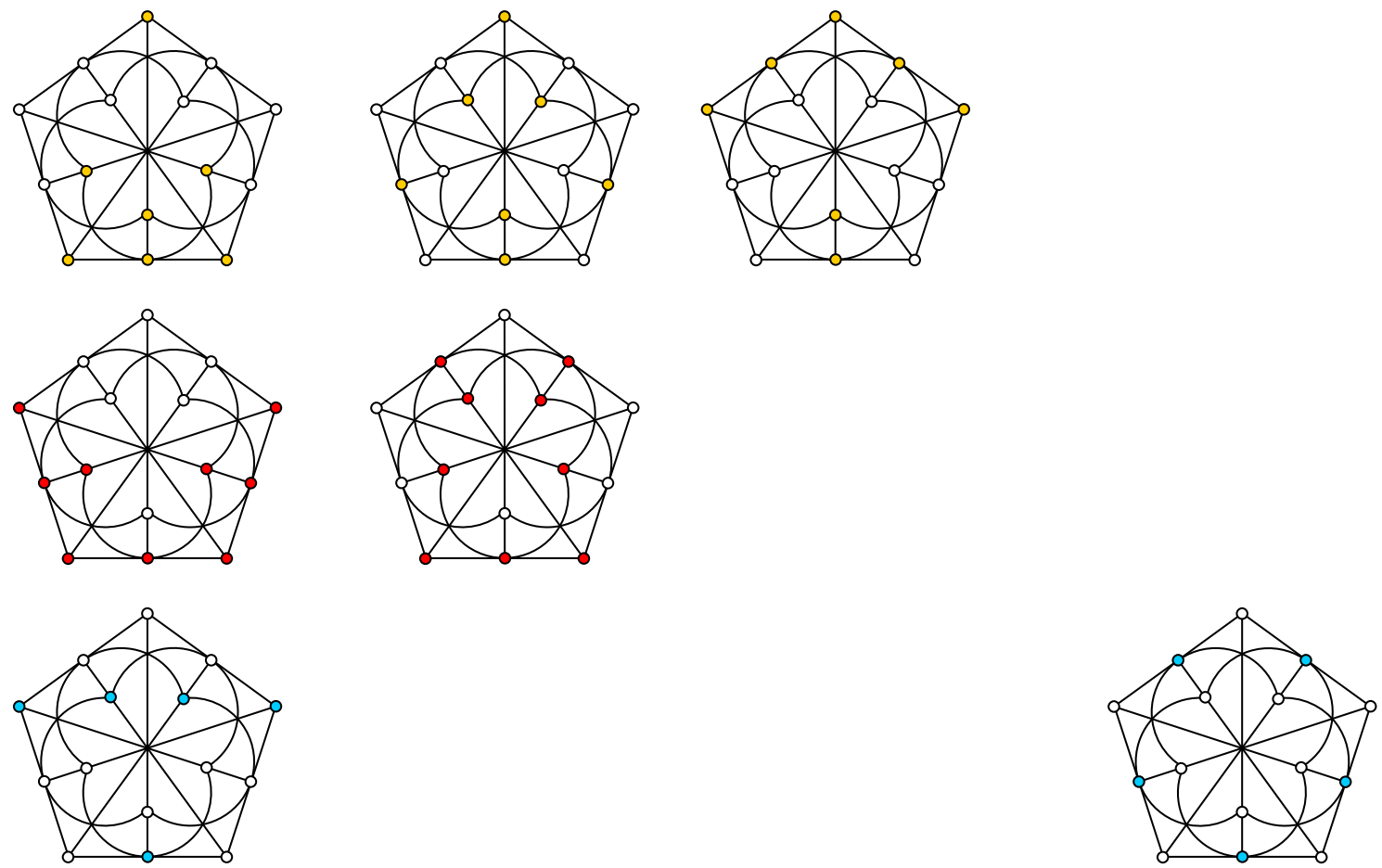

Figure 1. The three kinds of geometric hyperplanes of $W(2)$. The points of the quadrangle are represented by small circles and its lines are illustrated by the straight segments as well as by the segments of circles; note that not every intersection of two segments counts for a point of the quadrangle. The upper panel shows the points' perp-sets (yellow bullets), the middle panel grids (red bullets) and the bottom panel ovoids (blue bullets); the use of different colouring will become clear later. Each picture except that in the bottom right-hand corner - stands for five different hyperplanes, the four other being obtained from it by its successive rotations through 72 degrees around the center of the pentagon.
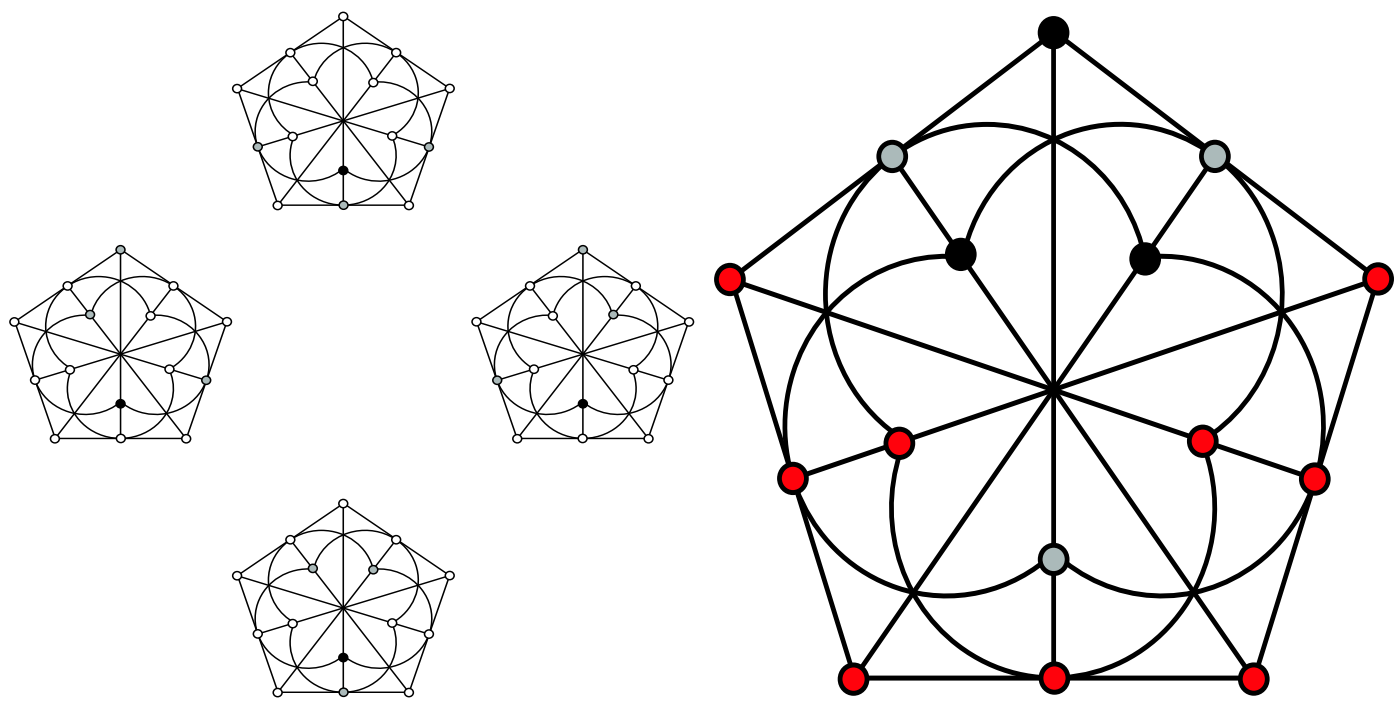

Figure 2. Left: - The four distinct unicentric triads (grey bullets) and their common center (black bullet); note that the triads intersect pairwise in a single point and their union covers fully the center's perp-set. Right: - A grid (red bullets) and its complement as a disjoint union of two complementary tricentric triads (black and grey bullets); the two triads are also seen to comprise a dual grid (of order $(1,2))$. 

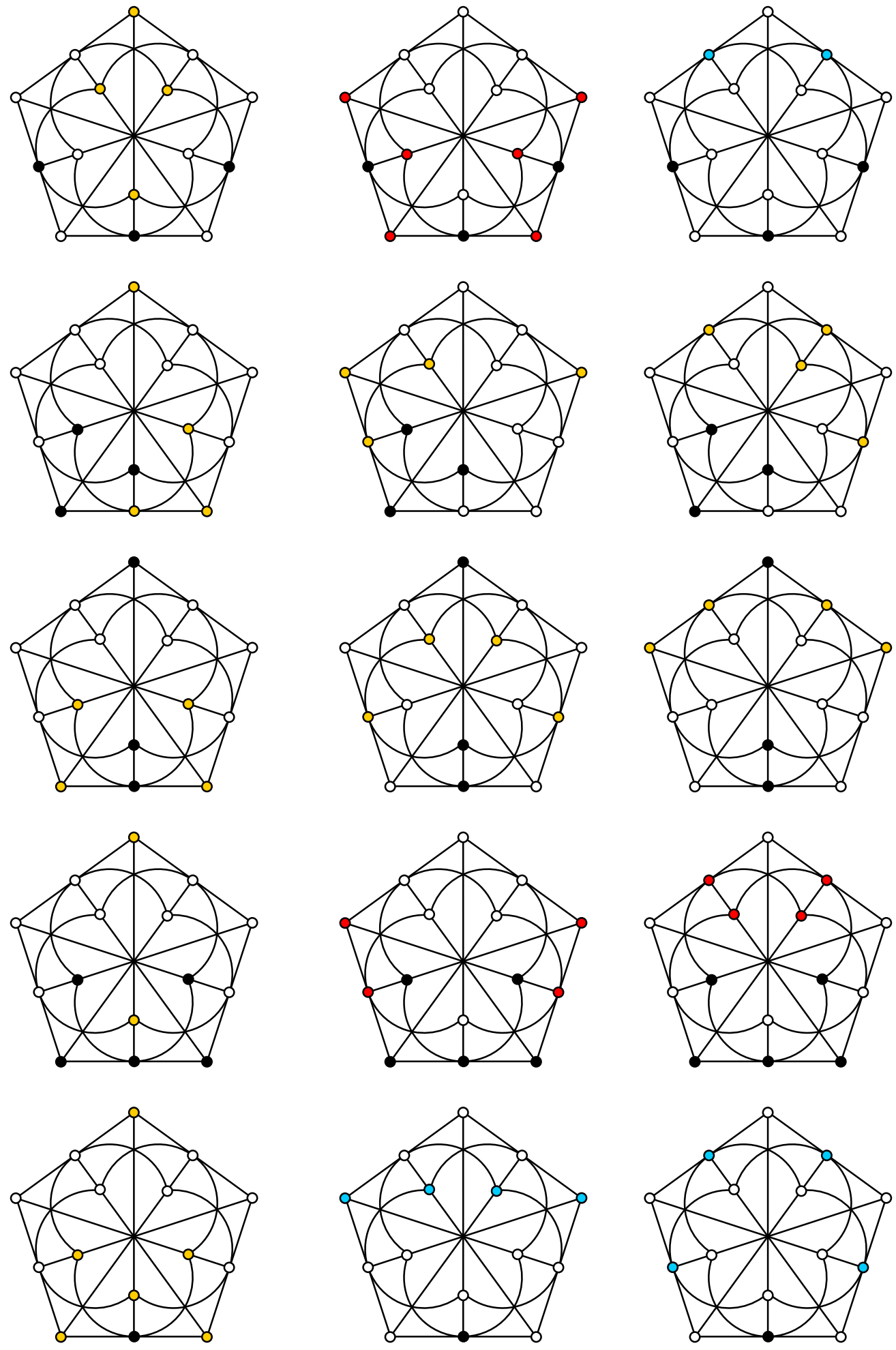

Figure 3. The five different kinds of the lines of $\mathcal{V}(W(2))$, each being uniquely determined by the properties of its core-set (black bullets). Note that the "yellow" hyperplanes (i.e., perp-sets) occur in each type, and yellow is also the colour of two homogeneous (i.e., endowed with only one kind of a hyperplane) types (2nd and 3rd row). It is also worth mentioning that the cardinality of core-sets is an odd number not exceeding five. The three hyperplanes of any line are always in such relation to each other that their union comprises all the points of $W(2)$. 
the number of such triads is $4 \times 15=60$. Tricentric triads always come in "complementary" pairs, one representing the centers of the other, and each such pair is the complement of a grid of $W(2)$ (Fig. 2, right); hence, the number of such triads is $2 \times 10=20$. A unicentric triad is always a subset of an ovoid, which is never the case for a tricentric triad; the latter, in graphcombinatorial terms, representing a complete bipartite graph on six vertices. Now, we have enough background information at hand to reveal the structure of the Veldkamp space of our "doily", $\mathcal{V}(W(2))^{2}$

From the definition given in Section 2, we easily see that $\mathcal{V}(W(2))$ consists of 31 points of which 15 are represented/generated by single-point perp-sets, 10 by grids and six by ovoids. The lines of $\mathcal{V}(W(2))$ feature three points each and are of five distinct types, as illustrated in Fig. 3. These types differ from each other in the cardinality and structure of "core-sets", i.e., the sets of points of $W(2)$ shared by all the three hyperplanes forming a given line. As it is obvious from Fig. 3, the lines of the first three types (the first three rows of the figure) have the core-sets of the same cardinality, three, differing from each other only in the structure of these sets as being unicentric triads, tricentric triads and triples of collinear points, respectively. The lines of the fourth type have as core-sets pentads of points, each being a quadruple of points collinear with a given point of $W(2)$, whereas core-sets of the last type's lines feature just a single point. A much more interesting issue is the composition of the lines. Just a brief look at Fig. 3 reveals that geometric hyperplanes of only one kind, namely perp-sets, are present on each line of $\mathcal{V}(W(2))$; grids and ovoids occur only on two kinds of the lines. We also see that the purely homogeneous types are those whose core-sets feature collinear triples and tricentric triads, the most heterogeneous type - the one exhibiting all the three kinds of hyperplanes - being that characterized by unicentric triads. We also notice that there are no lines comprising solely grids and/or solely ovoids, nor the lines featuring only grids and ovoids, which seems to be connected with the fact that the cardinality of a core-set is an odd number. From the properties of $W(2)$ and its triads as discussed above it readily follows that the number of the lines of type one to five is $60,20,15,45$ and 15, respectively, totalling 155. All these observations and facts are gathered in Table 1. We conclude this section with the observation that $\mathcal{V}(W(2))$ has the same number of points (31) and lines (155) as $P G(4,2)$, the four-dimensional projective space over the Galois field of two elements [8]; this is not a coincidence, as the two spaces are, in fact, isomorphic to each other [5].

\section{Pauli operators of two-qubits in light of $\mathcal{V}(W(2))$}

As discovered in [1] (see also [3]), the fifteen generalized Pauli operators/matrices associated with the Hilbert space of two-qubits (see, e.g., [9]) can be put into a one-to-one correspondence with the fifteen points of the generalized quadrangle $W(2)$ in such a way that their commutation algebra is completely and uniquely reproduced by the geometry of $W(2)$ in which the concept commuting/non-commuting translates into that of collinear/non-collinear. Given this mapping, it was possible to ascribe a definitive geometrical meaning to sets of three pairwise commuting generalized Pauli operators in terms of lines of $W(2)$ and to other three kinds of distinguished subsets of the operators having their counterparts in geometric hyperplanes of $W(2)$ as shown in Table 2 (see $[1,3]$ for more details). Yet, $\mathcal{V}(W(2))$ puts this bijection in a different light, in which other three subsets of the Pauli operators come into play, namely those represented by the two types of a triad and by the specific pentads occurring as the core-sets of the lines of $\mathcal{V}(W(2)$ ) (Table 1). As already mentioned, the role of tricentric triads of the operators has

\footnotetext{
${ }^{2}$ As this paper is primarily aimed at physicists rather than mathematicians, in what follows we opt for an elementary and self-contained exposition of the Veldkamp space of $W(2)$; this explanation is based only upon some very simple properties of $W(2)$ readily to be grasped from its depiction as "the doily", and does not presuppose/require any further background from the reader.
} 
Table 1. A succinct summary of the properties of the five different types of the lines of $\mathcal{V}(W(2))$ in terms of the core-sets and the types of geometric hyperplanes featured by a generic line of a given type. The last column gives the total number of lines per the corresponding type.

\begin{tabular}{|l|ccc|c|}
\hline \hline Type of Core-Set & Perp-Sets & Grids & Ovoids & $\#$ \\
\hline Single Point & 1 & 0 & 2 & 15 \\
Collinear Triple & 3 & 0 & 0 & 15 \\
Unicentric Triad & 1 & 1 & 1 & 60 \\
Tricentric Triad & 3 & 0 & 0 & 20 \\
Pentad & 1 & 2 & 0 & 45 \\
\hline \hline
\end{tabular}

Table 2. Three kinds of the distinguished subsets of the generalized Pauli operators of two-qubits (PO) viewed as the geometric hyperplanes in the generalized quadrangle of order two $(G Q)[1,3]$.

\begin{tabular}{llll}
\hline \hline PO & $\begin{array}{l}\text { set of five mutually } \\
\text { non-commuting operators }\end{array}$ & $\begin{array}{l}\text { set of six operators } \\
\text { commuting with a given one } \\
\text { gerp-set } \backslash\{\text { reference point }\}\end{array}$ & $\begin{array}{l}\text { nine operators of } \\
\text { ovoid }\end{array}$ \\
\hline \hline
\end{tabular}

been recognized in disguise of complete bipartite graphs on six vertices [3]. A true novelty here is obviously unicentric triads and pentads of the generalized Pauli operators as these are all intimately connected with single-point perp-sets; given a point of $W(2)$ (i.e., a generalized Pauli operator of two-qubits), its perp-set fully encompasses four unicentric triads (Fig. 2, left) and three pentads (Fig. 3, 4th row) of points/operators. This feature has also a very interesting aspect in connection with the conjecture relating the existence of mutually unbiased bases and finite projective planes raised in [10], because with each point $x$ of $W(2)$ there is associated a projective plane of order two (the Fano plane) whose points are the elements of $x^{\perp}$ and whose lines are the spans $\{u, v\}^{\perp \perp}$, where $u, v \in x^{\perp}$ with $u \neq v$ [4].

Identifying the Pauli operators of a two-qubit system with the points of the generalized quadrangle of order two led to the discovery of three distinguished subsets of the operators in terms of geometric hyperplanes of the quadrangle. Here we go one level higher, and identifying these subsets with the points of the associated Veldkamp space leads to recognition of nother remarkable subsets of the Pauli operators, viz. unicentric triads and pentads. It is really intriguing to see that these are the core-sets of the two kinds of lines that both feature grids alias Mermin squares. As it is well known, Mermin squares, which reveal certain important aspects of the entanglement of the system, play a crucial role in the proof of the Kochen-Specker theorem in dimension four and our approach gives a novel geometrical meaning to this [3, 11]. At the Veldkamp space level it turns out of particular importance to study relations between eigenvectors of the above-mentioned unicentric triads and pentad of operators in order to reveal finer, hitherto unnoticed traits of the structure of Mermin squares. These seem to be intimately connected with the existence of outer automorphisms of the symmetric group on six letters, which is the full group of automorphisms of our quadrangle; as this group is the only symmetric group possessing (non-trivial) outer automorphisms, this implies that two-qubits have a rather special footing among multiple qubit systems. All these aspects deserve special attention and will therefore be dealt with in a separate paper.

Concerning three-qubits, our preliminary study indicates that the corresponding finite geometry differs fundamentally from that of $W(2)$ in the sense that it contains multi-lines, i.e., two or more lines passing through two distinct points [12]. As we do not have a full picture at hand yet, we cannot see if it admits hyperplanes and so lends itself to constructing the corresponding Veldkamp space. If the latter does exist, it is likely to differ substantially from that of twoqubits, which would imply the expected difference between entanglement properties of the two 
kinds of systems; if not, this will only further strengthen the above-mentioned uniqueness of two-qubits.

\section{Conclusion}

By employing the concept of the Veldkamp space of the generalized quadrangle of order two, we were able to recognize other, on top of those examined in $[1,2,3]$, distinguished subsets of generalized Pauli operators of two-level quantum systems, namely unicentric triads and pentads of them. It may well be that these two kinds of subsets of the two-qubit Pauli operators hold an important key for getting deeper insights into the nature of finite geometries underlying multiple higher-level quantum systems $[12,13]$, in particular when the dimension of Hilbert space is not a power of a prime [14].

\section{Acknowledgements}

This work was partially supported by the Science and Technology Assistance Agency under the contract \# APVT-51-012704, the VEGA grant agency projects \# 2/6070/26 and \# 7012 (all from Slovak Republic), the trans-national ECO-NET project \# 12651NJ "Geometries Over Finite Rings and the Properties of Mutually Unbiased Bases" (France), the CNRS-SAV Project \# 20246 "Projective and Related Geometries for Quantum Information" (France/Slovakia) and by the 〈Action Austria-Slovakia〉 project \# 58s2 "Finite Geometries Behind Hilbert Spaces".

\section{References}

[1] Saniga M., Planat M., Pracna P., Projective ring line encompassing two-qubits, Theor. Math. Phys., to appear, quant-ph/0611063.

[2] Saniga M., Planat M., Multiple qubits and symplectic polar spaces of order two, Adv. Studies Theor. Phys. 1 (2007) 1-4, quant-ph/0612179.

[3] Planat M., Saniga M., On the Pauli graph of $N$-qudits, quant-ph/0701211.

[4] Payne S.E., Thas J.A., Finite generalized quadrangles, Pitman, Boston - London - Melbourne, 1984.

[5] Buekenhout F., Cohen A.M., Diagram geometry (Chapter 10.2), Springer, New York, to appear; preprints of separate chapters can be found at http://www.win.tue.nl/ amc/buek/.

[6] Ronan M.A., Embeddings and hyperplanes of discrete geometries, European J. Combin. 8 (1987), $179-185$.

[7] Shult E., On Veldkamp lines, Bull. Belg. Math. Soc. 4 (1997), 299-316.

[8] Hirschfeld J.W.P., Thas J.A., General Galois geometries, Oxford University Press, Oxford, 1991.

[9] Lawrence J., Brukner Č., Zeilinger A., Mutually unbiased binary observable sets on $N$ qubits, Phys. Rev. A 65 (2002), 032320, 5 pages, quant-ph/0104012.

[10] Saniga M., Planat M., Rosu H., Mutually unbiased bases and finite projective planes, J. Opt. B: Quantum Semiclass. Opt. 6 (2004), L19-L20, math-ph/0403057.

[11] Saniga M., Planat M., Minarovjech M., Projective line over the finite quotient $\operatorname{ring} G F(2)[x] /\left(x^{3}-x\right)$ and quantum entanglement: the Mermin "magic" square/pentagram, Theor. Math. Phys. 151 (2007), 625-631, quant-ph/0603206.

[12] Planat M., Saniga M., Pauli graph and finite projective lines/geometries, Optics and Optoelectronics, to appear, quant-ph/0703154.

[13] Thas K., Pauli operators of $N$-qubit Hilbert spaces and the Saniga-Planat conjecture, Chaos Solitons Fractals, to appear.

[14] Thas K., The geometry of generalized Pauli operators of N-qudit Hilbert space, Quantum Information and Computation, submitted. 